APPLICATIONES MATHEMATICAE

27,4 (2000), pp. $489-498$

O. HERNÁNDEZ-LERMA (México)

J. B. LASSERRE (Toulouse)

\title{
ON THE CLASSIFICATION OF MARKOV CHAINS VIA OCCUPATION MEASURES
}

Abstract. We consider a Markov chain on a locally compact separable metric space $X$ and with a unique invariant probability. We show that such a chain can be classified into two categories according to the type of convergence of the expected occupation measures. Several properties in each category are investigated.

1. Introduction. Consider a Markov chain $\left\{\Phi_{t}: t=0,1, \ldots\right\}$ on $(X, \mathcal{B})$ (with $X$ a locally compact separable metric space and $\mathcal{B}$ its usual Borel $\sigma$ field), with stochastic kernel $P$, and a unique invariant probability measure $\mu$ on $\mathcal{B}$. One way to characterize such a chain is to use the various notions of transience and recurrence as is done for instance in Meyn and Tweedie [7]. However, except for $X$ countable, there is no definite classification.

The aim of this paper is to show that according to the type of convergence of the sequence of expected occupation measures, those chains can be classified in only two categories. Namely, there is an absorbing set $A$ of full measure $\mu$ such that either (a) or (b) below occur:

(a) The chain is positive recurrent in $A$, and positive Harris recurrent in $H \subset A$ with $\mu(H)=1$; the sequence of expected occupation measures $\left\{P^{(n)}(x, \cdot)\right\}$ defined in $(2.1)$ converges to $\mu$ in the total variation norm for every $x \in H$. The set $H$ is a "maximal" Harris set and is indecomposable into disjoint absorbing sets.

2000 Mathematics Subject Classification: Primary 28A33; Secondary 28C15.

Key words and phrases: measures, setwise convergence, positive Harris recurrence, absolute continuity.

Research partially supported by the CNRS (France) - CONACYT (Mexico) Scientific Cooperation Program, and by the ECOS (France) - ANUIES (Mexico) Educational and Scientific Cooperation Program. The research of the first author was also supported by CONACYT Grant 32299-E. 
(b) The chain is not positive recurrent and for every $x \in A$, the sequence $\left\{P^{(n)}(x, \cdot)\right\}$ converges to $\mu$ only weakly (and the stronger setwise convergence or in the total variation norm are not possible). In addition, $A$ can be decomposed into an uncountable collection of $\mu$-null absorbing sets $A_{x} \subset A$, that is, when the chain starts in state $x \in A$, it remains in a $\mu$-null absorbing set $A_{x}$. The restriction of $P$ to $A_{x}$ has no invariant probability measure. Thus, in contrast to case (a), the absorbing set $A$ can be further decomposed into uncountably many absorbing sets.

In addition, a solidarity property holds. Namely, if the convergence of $P^{(n)}(x, \cdot)$ to $\mu$ in total variation occurs on a $\mu$-positive set then it occurs $\mu$-a.e. and we are in case (a). In fact, to be in case (a), it even suffices to check whether the sequence $P^{(n)}(x, O)$ converges for all open sets $O \in \mathcal{B}$ on a $\mu$-positive set only (note that knowledge of $\mu(O)$ is not required).

Moreover, in case (b), for every bounded measurable function $f$ on $X$ and for every $x \in A$, whenever the limit $f^{*}(x)$ in the Birkhoff Individual Ergodic Theorem exists, it is shown to be $\int f d \theta_{x}$ for some pure finitely additive invariant probability $\theta_{x}$ and $\int f d \mu=\int f d \theta_{x}$ for $\mu$-a.a. $x$. As $A$ can be partitioned into $\mu$-null absorbing sets $A_{x}$, the restriction of $P$ to $A_{x}$ is still a Markov chain but with no $P$-invariant probability measure. Still, the limit in the Birkhoff Individual Ergodic Theorem has an integral representation, but now with respect to a finitely additive probability.

One may regard case (a) as the analogue of the countable case, i.e., the case of an irreducible Markov chain where $A$ cannot be decomposed into absorbing sets, wheras case (b) illustrates what can happen if $X$ is not countable. The latter case is far from being pathological. In fact, one may show that every Markov chain with finitely supported expected occupation measures, and whose invariant distribution does not have a countable support, will fall into case (b). This can be checked easily in many cases. For instance, as shown in some examples, many measure-preserving transformations in $\mathbb{R}^{n}$, random dynamical systems with finitely supported noise-distribution (among them, random walks with finite distributions) and iterated functions systems will fall into this class. For such systems (with a unique invariant probability measure $\mu$ ), for $\mu$-a.a. initial states $x$, the chain evolves in a countable space $A_{x}$ and the associated Markov chain (the restriction to $A_{x}$ ) has no invariant probability measure.

Finally, it is important to note that for the purpose of this paper, the uniqueness assumption is not restrictive. Indeed, in the case of a non-unique invariant probability measure, and thanks to an ergodic decomposition à la Yosida, one may restrict to the ergodic classes in that decomposition in which there is a unique invariant probability measure (see e.g. [3]). 
2. Notation and definitions. With $(X, \mathcal{B})$ a measurable space, let $M(X)$ be the Banach space of finite signed measures on $\mathcal{B}$, endowed with the total variation norm $\|\cdot\|$, whereas $L_{1}(\mu)$ denotes the standard Banach space $L_{1}(X, \mathcal{B}, \mu)$.

Let $P(x, \cdot)$ be the transition kernel of a discrete-time homogeneous Markov chain $\Phi=\left\{\Phi_{t}: t=0, \ldots\right\}$ on $X$, i.e.,

- For every $x \in X, P(x, \cdot)$ is a probability measure (p.m.) on $\mathcal{B}$.

- For every $B \in \mathcal{B}, P(\cdot, B)$ is a measurable function on $X$.

Denote by $P^{(n)}(x, \cdot)$ the $n$-step expected occupation measure with initial state $x \in X$, i.e.,

$$
P^{(n)}(x, B):=n^{-1} \sum_{t=0}^{n-1} P^{t}(x, B), \quad B \in \mathcal{B} .
$$

The transition kernel $P$ defines a linear operator on $M(X), \mu \mapsto \mu P$, by

$$
\mu P(B):=\int P(x, B) \mu(d x), \quad B \in \mathcal{B} .
$$

A p.m. $\mu$ in $M(X)$ is called a $P$-invariant probability measure $(P$-invariant p.m.) if $\mu P=\mu$.

A set $B \in \mathcal{B}$ is called a $\mu$-null set if $\mu(B)=0$. A measure $\nu$ is said to be singular with respect to $\mu$ (denoted by $\nu \perp \mu$ ) if there is a set $A \in \mathcal{B}$ with $\mu(A)=0=\nu(X \backslash A)$.

3. Main results. In this section we first consider a measurable space $(X, \mathcal{B})$ with $\mathcal{B}$ countably generated. We use a result of Neveu [8] to classify into two categories the chains with a unique invariant p.m. (i.p.m.). Then, using a result from [4] that characterizes positive recurrent Harris chains in terms of the convergence of the expected occupation measures, we obtain a solidarity property (see Proposition 3.2 below). We then consider the case where $X$ is a locally compact separable metric space and $\mathcal{B}$ its usual Borel $\sigma$-field. In this case, the two categories of chains can be identified via the type of convergence of the expected occupation measures (Theorem 3.3). Moreover, a necessary and sufficient condition that only involves open sets permits one to detect to which category the chain belongs (Proposition 3.5).

3.1. General measurable space. We first have the following result that is an extension of Proposition 2 of Neveu [8] (see also Horowitz [5]).

Proposition 3.1. Assume that $P$ has a unique P-invariant p.m. $\mu$. Then there is an absorbing set $A \in \mathcal{B}$ with $\mu(A)=1$ and such that either

(a) $\Phi$ is positive recurrent in $A$ and positive Harris recurrent in $H \subset A$ with $\mu(H)=1$, or 
(b) $\mu \perp \sum_{i=1}^{\infty} P^{i}(x, \cdot)$ for each $x \in A$ so that when the chain $\Phi$ starts in state $x \in A$, it remains in a $\mu$-null absorbing set $A_{x} \subset A$. There are uncountably many such sets.

Of course, when $X$ is countable, only (a) can occur.

Proof. As $\mu$ is the unique $P$-invariant p.m., by the celebrated Birkhoff Individual Ergodic Theorem (see e.g. [11]), for all $B \in \mathcal{B}$ one has

$$
\lim _{n \rightarrow \infty} n^{-1} \sum_{i=1}^{n} P^{i}(x, B)=\mu(B) \quad \mu \text {-a.e. }
$$

Therefore, whenever $\mu(B)>0$,

$$
\sum_{i=1}^{\infty} P^{i}(x, B)>0 \quad \mu \text {-a.e. }
$$

which implies that $\Phi$ is $\mu$-essentially irreducible (see e.g. Neveu [8]). Therefore, from Proposition 2 of Neveu [8], only two things may happen. Either (a) there is an absorbing set $A_{1} \in \mathcal{B}$ with $\mu\left(A_{1}\right)=1$ such that

$$
\mu \ll \sum_{i=1}^{\infty} P^{i}(x, \cdot) \quad \text { for all } x \in A_{1},
$$

or (b) there is an absorbing set $A_{2} \in \mathcal{B}$ with $\mu\left(A_{2}\right)=1$ such that

$$
\mu \perp \sum_{i=1}^{\infty} P^{i}(x, \cdot) \quad \text { for all } x \in A_{2} .
$$

In case (a), the chain is $\mu$-irreducible in the absorbing set $A_{1}$, and, having a unique $P$-invariant p.m., it is positive recurrent (see e.g. Meyn and Tweedie [7]) in $A_{1}$. In addition, there is a set $H \in \mathcal{B}$ and a $\mu$-null set $N$ such that $A_{1}=H \cup N$, and $H$ is a maximal Harris set in which $\Phi$ is positive Harris recurrent (see e.g. Theorem 9.1.5 in [7]).

In case (b), it follows from (3.1) that for each $x \in A:=A_{2}$, there is a Borel set $A_{x} \subset A$ such that $\mu\left(A_{x}\right)=0$ and $P^{i}\left(x, A_{x}\right)=1$ for all $i=1,2, \ldots$ Thus, the $\mu$-null set $A_{x}^{\prime}:=\left\{y: P^{j}\left(y, A_{x}\right)=1\right.$ for $\left.j=1,2, \ldots\right\} \subset$ $A$ is absorbing and $x \in A_{x}^{\prime}$. As the $A_{x}^{\prime}$ form a covering of $A$, there are uncountably many different such sets $A_{x}^{\prime}$ because otherwise, $\mu\left(A_{x}^{\prime}\right)=0$ for all $x$ and $\mu(A)=1$ would yield a contradiction.

We now use Proposition 3.1 to obtain the following solidarity property:

Proposition 3.2. Assume that $\Phi$ has a unique P-invariant p.m. $\mu$. Then the following statements are equivalent:

(a) $P^{(n)}(x, \cdot) \rightarrow \mu$ setwise for all $x \in B \in \mathcal{B}$ with $\mu(B)>0$.

(b) $P^{(n)}(x, \cdot) \rightarrow \mu$ setwise $\mu$-a.e.

(c) $\left\|P^{(n)}(x, \cdot)-\mu\right\| \rightarrow 0 \mu$-a.e. 
Proof. The implications $(\mathrm{c}) \Rightarrow(\mathrm{b}) \Rightarrow(\mathrm{a})$ are trivial. The implication $(b) \Rightarrow(c)$ is proved in [4]. Therefore, it suffices to prove $(a) \Rightarrow(b)$.

If (a) holds and $B \in \mathcal{B}$ is such that $\mu(B)>0$, then on $B$ we cannot have $\mu \perp P^{k}(x, \cdot)$ for all $k=0,1, \ldots$ Therefore, by Proposition 3.1(a), the chain is positive recurrent in an absorbing set of full $\mu$-measure and positive Harris recurrent on some maximal Harris set $H \subset A$. From a result of [4], we conclude that $P^{(n)}(x, \cdot) \rightarrow \mu$ setwise for all $x \in H$ and in fact $\left\|P^{(n)}(x, \cdot)-\mu\right\| \rightarrow 0$ for all $x \in H$.

3.2. Locally compact separable metric space. In this section, we assume that $X$ is a locally compact separable metric space with $\mathcal{B}$ its usual Borel $\sigma$-field. In this case, whenever $P$ has a $P$-invariant p.m. $\mu$, there is a Yosida ergodic decomposition in the sense that there is a family of absorbing sets $\left\{E_{\alpha}\right\}$ called "ergodic classes" where the restriction of $\Phi$ to each $E_{\alpha}$ has a unique i.p.m. $\mu_{\alpha}$, and

$$
P^{(n)}(x, \cdot) \Rightarrow \mu_{\alpha} \quad \forall x \in E_{\alpha}
$$

(see e.g. [3]), where " $P^{(n)}(x, \cdot) \Rightarrow \mu_{\alpha}$ " denotes weak convergence. Therefore, we may now characterize the behavior of the chain in each ergodic class by reducing to the case of a unique $P$-invariant p.m.

Theorem 3.3. Assume that $\Phi$ has a unique P-invariant p.m. $\mu$. Then only (a) or (b) below may occur:

(a) $\Phi$ is positive recurrent (resp. positive Harris recurrent) in an absorbing set $A$ with $\mu(A)=1$ (resp. in an absorbing set $H \subset A$ with $\mu(H)=1$ ) and for $\mu$-a.a. $x \in X$ (resp. for all $x \in H$ ), one has

$$
\left\|P^{(n)}(x, \cdot)-\mu\right\| \rightarrow 0 .
$$

(b) $\Phi$ is not positive recurrent and for $\mu$-a.a. $x \in X$, one has

$$
P^{(n)}(x, \cdot) \Rightarrow \mu \quad \text { and } \quad \mu \perp P^{(n)}(x, \cdot) .
$$

Pro of. In the case of Proposition 3.1(a), the chain $\Phi$ is positive recurrent in an absorbing set $A$ with $\mu(A)=1$ and positive Harris recurrent in a maximal Harris set $H \subset A$. Therefore, by a result of [4], (3.2) holds in $H$. This yields (a) since $\mu(H)=1$. The case (b) follows from Proposition 3.1(b) and Theorem 3.1(b) of [3].

REMARK 3.4. Theorem 3.3 states that $\mu$-a.e., either the expected occupation measures converge in the strongest possible way (in the total variation norm), or "at most" the weakest form (the weak convergence " $\Rightarrow$ "). Nothing in-between can happen! For instance, we cannot have setwise convergence on some $\mu$-positive set $B \in \mathcal{B}$ and only weak convergence on some other $\mu$-positive set $C \in \mathcal{B}$. 
Thus, from the solidarity property in Proposition 3.2 it follows that to check which type of convergence occurs (in norm or only weak), it suffices to consider one $\mu$-positive set only.

In addition, we even have:

Proposition 3.5. Assume that $\Phi$ has a unique P-invariant p.m. $\mu$. Then:

(a) $\Phi$ is positive recurrent in an absorbing set of full measure if and only if there is some $\mu$-positive set $B \in \mathcal{B}$ such that $P^{(n)}(x, O)$ converges for every open set $O \in \mathcal{B}$ and every $x \in B$.

(b) $\Phi$ is not positive recurrent in any absorbing set if and only if there is some $\mu$-positive set $B \in \mathcal{B}$ such that for every $x \in B, P^{(n)}\left(x, O_{x}\right)$ does not converge for some open set $O_{x} \in \mathcal{B}$.

P r o of. It suffices to observe that the setwise convergence of a sequence of probability measures occurs if and only if it occurs in the class of open sets only (see Corollary 1 in Panchapagesan [9], which is an extension of Dieudonné's result in the compact case).

Observe that in (a), one does not need to know the limit which is $\mu(O)$. It suffices that the sequence $P^{(n)}(x, O)$ converges.

3.3. Examples. In this section, we provide a series of examples.

Measure-preserving transformations. Consider the following measurepreserving transformations (see Lasota and Mackey [6]):

(a) The $r$-adic transformation $S:[0,1] \rightarrow[0,1]$ given by $x \mapsto r x(\bmod 1)$ with $r>0$.

(b) The Ulam-von Neumann transformation $S:[0,1] \rightarrow[0,1]$ given by $x \mapsto S(x):=4 x(1-x)$.

(c) The Baker transformation $S:[0,1]^{2} \rightarrow[0,1]^{2}$ given by

$$
(x, y) \mapsto S(x, y)= \begin{cases}(2 x, y / 2), & 0 \leq x \leq 1 / 2,0 \leq y \leq 1, \\ (2 x-1,(y+1) / 2), & 1 / 2 \leq x \leq 1,0 \leq y \leq 1 .\end{cases}
$$

(d) Anosov diffeomorphisms $S:[0,1]^{2} \rightarrow[0,1]^{2}$ given by

$$
(x, y) \mapsto S(x, y)=(x+y, x+2 y)(\bmod 1) .
$$

In cases (a), (c) and (d), the Lebesgue measure is invariant, whereas in case (b), there is an invariant measure with density $(\pi \sqrt{x(1-x)})^{-1}$.

Therefore, since in all cases the expected occupation measures are finitely supported, the convergence of the expected occupation measures to the invariant probability $\mu$ is only "weak" and therefore, the corresponding Markov chains cannot be positive recurrent, that is, we are in case (b) of Theorem 3.3. 
Systems $x_{t+1}=F\left(x_{t}, \xi_{t}\right), t=0,1, \ldots$, where $F: \mathbb{R}^{n} \rightarrow \mathbb{R}^{n}$ is measurable and the $\xi_{t}$ are i.i.d. $n$-random vectors. If $\xi_{0}$ takes only a finite number of values and the invariant measure (assumed to exist and to be unique) is not discrete, then the chain cannot be positive recurrent and we are in case (b) of Theorem 3.3. Most random walks with finite distributions fall into that class.

Barnsley "Iterated Function Systems". Consider $m$ mappings $F_{i}$ : $\mathbb{R}^{n} \rightarrow \mathbb{R}^{n}$, and the system

$$
x_{t+1}=F_{\xi_{n}}\left(x_{t}\right), \quad t=0,1, \ldots,
$$

where the $\xi_{t}$ are i.i.d. random variables with values in $\{1, \ldots, m\}$ and distribution $\left[p_{1}, \ldots, p_{m}\right]$. Under certain conditions, the induced Markov chain has a unique absolutely continuous i.p.m., called a fractal measure (a measure supported on a fractal); see e.g. Section 12.8 in Lasota and Mackey [6]. Again, as the expected occupation measures are finitely supported, the chain cannot be positive recurrent and the convergence is only weak.

4. On the Birkhoff Individual Ergodic Theorem. In this section, $(X, \mathcal{B})$ is again a measurable space with $\mathcal{B}$ countably generated. From the results of the previous section, in the case of a unique i.p.m. $\mu$ and when the chain is not positive recurrent, for $\mu$-almost all initial states, the chain remains in a $\mu$-null absorbing set $A_{x}$. Therefore, the restriction of the transition kernel to $A_{x}$ is still a Markov chain but with no i.p.m. However, the limit in the Birkhoff Ergodic Theorem is still related to the i.p.m. $\mu$. We make this more precise in Theorem 4.1 below.

Let $B(X)$ be the Banach space of bounded measurable functions on $X$, equipped with the sup-norm. From the Birkhoff Individual Ergodic Theorem (see e.g. [11]), we already know that for every $f \in L_{1}(\mu)$,

$$
P^{(n)} f(x) \rightarrow f^{*}(x) \quad \mu \text {-a.e. and } \int f d \mu=\int f^{*} d \mu .
$$

In fact, from the uniqueness of $\mu$ it follows that $f^{*}(x)=\int f d \mu$ for $\mu$-a.a. $x \in X$. Moreover, in the case that $\Phi$ is positive recurrent (resp. positive Harris recurrent), we also have

$$
\left\|P^{(n)}-\mu\right\| \rightarrow 0 \quad \mu \text {-a.e. } \quad(\text { resp. for all } x \in X)
$$

so that if $\Phi$ is positive recurrent, there is a set $A$ with $\mu(A)=1$ such that

$$
P^{(n)} f \rightarrow \int f d \mu \quad \forall x \in A, f \in B(X),
$$

and if $\Phi$ is Harris recurrent, one may replace $A$ with $X$. We now show that if $\Phi$ is not recurrent, then for every $x \in X, \lim _{n} P^{(n)} f(x)$ (whenever exists) can be written as $\int f d \varphi_{x}$ with $\varphi_{x}$ a purely finitely additive measure (called a pure mean) which is $P$-invariant. The link with $\mu$ is that for every 
$f \in B(X), \int f d \varphi_{x}=\int f d \mu, \mu$-a.e. Part (i) of Theorem 4.1 below does not depend on the uniqueness of the i.p.m. $\mu$.

Theorem 4.1. (i) For every $x \in X$, there is a finitely additive (probability) measure $\varphi_{x}$ such that whenever $f \in B(X)$ and $\lim _{n} P^{(n)} f(x)$ exists, we have

$$
\lim _{n \rightarrow \infty} P^{(n)} f(x)=\int f d \varphi_{x} .
$$

Moreover, for every P-invariant p.m. $\mu$ one has

$$
\mu(B)=\int \varphi_{x}(B) \mu(d x), \quad B \in \mathcal{B} .
$$

(ii) In addition, if $\Phi$ has a unique P-invariant p.m. $\mu$, then either (a) or (b) below occurrs:

(a) $\Phi$ is positive recurrent in an absorbing set $A$ with $\mu(A)=1$ (and positive Harris recurrent in a maximal Harris set $H \subset A)$, and $\varphi_{x}=$ $\mu, \mu$-a.e. $($ for all $x \in H)$,

(b) $\Phi$ is not positive recurrent, and $\mu$-a.e., $\varphi_{x}$ is a purely finitely additive $P$-invariant probability; for every $f \in B(X), \int f d \mu=\int f d \varphi_{x}$ for p-a.a. $x \in X$.

P r o of. (i) The proof of the first statement is an application of a special Hahn-Banach Extension Theorem (Proposition 5, p. 224 of Royden [10]). Indeed, choose an arbitrary $x \in X$, and with $f^{*}(x)$ as in (4.1), let

$$
V_{x}:=\left\{f \in B(X): f^{*}(x) \text { exists }\right\} .
$$

Then $V_{x}$ is a nonempty subspace of $B(X)$ since it contains at least all the constant functions $f:=c$ for which $f^{*}(x)=c$. In addition,

$$
\left|f^{*}(x)\right| \leq\|f\| \quad \forall f \in V_{x}, \quad \text { and } \quad f \in V_{x} \Rightarrow P f \in V_{x},
$$

so that $P V_{x} \subset V_{x}$. Let $T_{x}: V_{x} \rightarrow \mathbb{R}$ be the linear mapping

$$
f \mapsto T_{x} f:=f^{*}(x) \quad \text { for } f \in V_{x} .
$$

Observe that $\left\{I, P, P^{2}, \ldots\right\}$ is an Abelian semigroup of linear operators on $B(X)$. Therefore, as $\|P f\| \leq\|f\|$ for all $f \in B(X)$, and in view of (4.6), from the Hahn-Banach Extension Theorem $T_{x}$ can be extended to all of $B(X)$, that is, to a continuous linear functional on $B(X)$, which we denote by $T_{x}$ again. As $1 \in V_{x}$ and $T_{x}$ is positive on $V_{x}, T_{x}$ can be chosen to be a positive linear functional. Hence, $T_{x}$ can be identified with a (nonnegative) bounded linear functional $\varphi_{x}$ on $\mathcal{B}$ that satisfies:

- $T_{x} f=\int f d \varphi_{x}$ for all $f \in B(X)$.

- $f^{*}(x)=T_{x} f=\int f d \varphi_{x}$ for all $f \in V_{x}$.

- $T_{x}(P f)=T_{x} f$ for all $f \in B(X)$. 
Therefore, $\varphi_{x} P=\varphi_{x}$, i.e., $\varphi_{x}$ is $P$-invariant. In addition, being finitely additive, $\varphi_{x}$ can be written as

$$
\varphi_{x}=\nu_{x}+\psi_{x}, \quad x \in X
$$

where $\nu_{x}$ is a countably additive measure and $\psi_{x}$ a a purely finitely additive measure. Finally, using an argument due to Neveu and proceeding as in Foguel [2], one may show that both $\nu_{x}$ and $\psi_{x}$ are $P$-invariant.

We now prove the second statement of (i). Let $\mu$ be a $P$-invariant p.m. and take an arbitrary $B \in \mathcal{B}$. From the Birkhoff Individual Ergodic Theorem with $f:=I_{B} \in L_{1}(\mu)$, one has $I_{B}^{*}(x)=\lim _{n} P^{(n)} f(x) \mu$-a.e. and $\int f^{*} d \mu=$ $\int f d \mu=\mu(B)$. Now, as $I_{B}^{*}(x)=\int I_{B} d \varphi_{x}=\varphi_{x}(B) \mu$-a.e., one may extend $\varphi_{\bullet}(B)$ so as to be measurable and, therefore,

$$
\int f^{*} d \mu=\int \varphi_{x}(B) \mu(d x)=\mu(B), \quad B \in \mathcal{B} .
$$

(ii) From Theorem 3.3, only two cases may occur:

(a) $\Phi$ is positive recurrent in an absorbing set $A$ with $\mu(A)=1$ and positive Harris recurrent in a maximal Harris set $H \subset A$. In addition, $\mu$-a.e. $\left\|P^{(n)}(x, \cdot)-\mu\right\| \rightarrow 0$ so that obviously, $\varphi_{x}=\mu \mu$-a.e. (and for all $x \in H$ ).

(b) $\Phi$ is not recurrent and from Proposition 3.1, for $\mu$-a.a. $x \in X$, $\mu \perp \sum_{i=1}^{\infty} P^{i}(x, \cdot)$ so that $\mu$-a.e.,

$$
P^{(n)}(x, \cdot) \perp \mu \quad \forall n=1,2, \ldots
$$

Therefore, for $\mu$-a.a. $x \in X$, there is a set $A_{x} \in \mathcal{B}$ with $\mu\left(A_{x}\right)=1$ and $P^{(n)}\left(x, A_{x}\right)=0$ for all $n=1,2, \ldots$ From the decomposition $\varphi_{x}=\nu_{x}+\psi_{x}$, the invariance of $\nu_{x}$ and the uniqueness of $\mu$, it follows that $\nu_{x}=\alpha_{x} \mu$ for some scalar $0 \leq \alpha_{x} \leq 1$. But then

$$
0=\lim _{n \rightarrow \infty} P^{(n)}\left(x, A_{x}\right)=\alpha_{x} \mu\left(A_{x}\right)+\psi_{x}\left(A_{x}\right)=\alpha_{x}+\psi_{x}\left(A_{x}\right),
$$

which implies that $\alpha_{x}=0$ and thus, for $\mu$-a.a. $x \in X, \varphi_{x}\left(=\psi_{x}\right)$ is a purely finitely additive measure. In addition, from Birkhoff's Individual Ergodic Theorem, since $f \in L_{1}(\mu)$ if $f \in B(X)$, and with $f^{*}$ as in (4.1), we have $f^{*}(x)=\int f d \varphi_{x}$ for $\mu$-almost all $x \in X$, and thus since we also have $f^{*}(x)=\int f d \mu$, we obtain $\int f d \mu=\int f d \varphi_{x}$ for $\mu$-almost all $x \in X$.

\section{References}

[1] J. L. Doob, Measure Theory, Grad. Texts in Math. 143, Springer, New York, 1994.

[2] S. R. Foguel, The Ergodic Theory of Markov Processes, Van Nostrand Math. Stud. 21, Van Nostrand, London, 1969.

[3] O. Hernández-Lerma and J. B. Lasserre, Ergodic theorems and ergodic decompostion for Markov chains, Acta Appl. Math. 54 (1998), 99-119. 
[4] O. Hernández-Lerma and J. B. Lasserre, Further criteria for positive Harris recurrence of Markov chains, Proc. Amer. Math. Soc., to appear.

[5] S. Horowitz, Transition probabilities and contractions of $L_{\infty}$, Z. Wahrsch. Verw. Gebiete 24 (1972), 263-274.

[6] A. Lasota and M. C. Mackey, Chaos, Fractals and Noise: Stochastic Aspects of Dynamics, Appl. Math. Sci. 97, Springer, New York, 1994.

[7] S. P. Meyn and R. L. Tweedie, Markov Chains and Stochastic Stability, Springer, London, 1993.

[8] J. Neveu, Sur l'irréductibilité des chaînes de Markov, Ann. Inst. H. Poincaré 8 (1972), 249-254.

[9] T. V. Panchapagesan, Baire and $\sigma$-Borel characterizations of weakly compact sets in $M(T)$, Trans. Amer. Math. Soc. 350 (1999), 4839-4847.

[10] H. L. Royden, Real Analysis, 3rd ed., Macmillan, New York, 1988.

[11] K. Yosida, Functional Analysis, 6th ed., Grundlehren Math. Wiss. 123, Springer, Berlin, 1980.

Onésimo Hernández-Lerma

Departamento de Matemáticas

CINVESTAV-IPN

Apdo. Postal 14-740

México, D.F. 07000, Mexico

E-mail: ohernand@math.cinvestav.mx
Jean B. Lasserre

LAAS-CNRS

7 Avenue du Colonel Roche 31077 Toulouse Cedex, France E-mail: lasserre@laas.fr 